\title{
REABILITAÇÃO ESTÉTICA COM PREPARO MINIMAMENTE INVASIVO: relato de caso
}

\section{AESTHETIC REHABILITATION USING A MINIMALLY INVASIVE APPROACH: a case report.}

\author{
Ludmilla Ribeiro Lopes ${ }^{1}$; Rita de Cássia Rocha Oliveira Souza ${ }^{1}$; Camila Maria \\ Martins Brandão ${ }^{2}$; Eurielva Saraiva de Sousa Oliveira ${ }^{3}$; Eduardo Buozi Moffa ${ }^{4}$
}

RESUMO: Devido à conscientização da sociedade, quanto á importância da saúde bucal e da estética, a busca por um sorriso bonito e harmônico tem sido crescente, aumentando cada vez mais a procura por procedimentos restauradores eficientes e com resultados favoráveis. O profissional da odontologia deve entender que a escolha dos materiais e técnicas utilizadas implicará no resultado final do tratamento. Em função dos seus resultados previsíveis e da conservação da estrutura do dente, os laminados cerâmicos são indicados para o tratamento estético de dentes anteriores. As cerâmicas têm se tornado alternativa para essas reabilitações de alta exigência estética, uma vez que possuem propriedades como biocompatibilidade, estabilidade de cor, longevidade e resistência, ou seja, apresentam biomimetismo com esmalte. Dessa forma, esse relato de caso tem como objetivo destacar as etapas na reabilitação oral estética usando laminados cerâmicos de mínima espessura com preparos minimamente invasivos. A metodologia para esta finalidade será um estudo exploratório, descritivo, como relato de caso clínico. A técnica escolhida foi de reanatomização com lentes de contatos. Ao final da intervenção clínica, foi obtido um resultado satisfatório, onde a função e a estética esperadas pelo paciente foram alcançadas.

PALAVRAS-CHAVE: Reabilitação estética anterior; Lentes de contato; Laminados cerâmicos.

\footnotetext{
1 Alunas de Graduação do curso de Odontologia, Universidade CEUMA.E-mail: ludy1203@ hotmail.com e ritadecassia123@hotmail.com

22Aluna de Pós-graduação em Odontologia, Universidade CEUMA. E-mail: camilammbrandao@ hotmail.com

3 Professora Mestre em Ciências da Saúde do Curso de Graduação em Odontologia, Universidade

CEUMA.E-mail: eurielva@terra.com.br

4 Professor Doutor em Reabilitação Oral dos Programas de Graduação em Odontologia e Pós-Graduação em Odontologia e Biologia Parasitária, Universidade CEUMA. E-mail: du_moffa@yahoo.com.br
} 
Revista Ceuma Perspectivas, Edição Especial, V Congresso de Saúde e Bem Estar Ceuma. Vol. 30, n02, 2017. ISSN Eletrônico: 2525-5576.

\begin{abstract}
Due to society's awareness of the importance of oral health and aesthetics, the search for a beautiful and harmonious smile has been increasing, increasing the search for efficient restorative procedures with favorable results. The dental professional must understand that the choice of materials and techniques used will imply the final result of the treatment. Due to its predictable results and the preservation of the tooth structure, ceramic laminate veneers, also called as "contact lenses", are indicated for the aesthetic treatment of anterior teeth. Ceramics have become an alternative to these highly esthetic restorations, since they have properties such as biocompatibility, color stability, longevity and resistance, that is, they exhibit biomimetism with enamel. Thus, this case report aims to highlight the steps in oral aesthetic rehabilitation using minimal thickness ceramic laminates with minimally invasive preparations. It was performed an exploratory descriptive case report. The technique chosen was teeth reanatomization using all ceramic veneers. As a final outcome at the end of the clinical procedures the expectations in regard to the new esthetic pattern of the patient were successfully reached.
\end{abstract}

KEYWORDS: anterior aesthetic rehabilitation; contact lens porcelain veneers; laminated ceramic veneers. 
Revista Ceuma Perspectivas, Edição Especial, V Congresso de Saúde e Bem Estar Ceuma. Vol. 30, n02, 2017. ISSN Eletrônico: 2525-5576.

\section{INTRODUÇÃO}

A odontologia adesiva teve inicio quando Buonocore, em 1955, proporcionou à dentística o condicionamento ácido do esmalte, dessa forma então solucionando o maior problema da dentística da época que era a falta de adesão dos materiais restauradores (BUONOCORE,1955).A partir dos estudos de Simonsen \& Calamia, (1987), os laminados cerâmicos foram consideradas como um importante recurso estético, em função da adesão entre o esmalte e facetas laminadas de porcelana condicionadas em sua face interna com ácido hidrofluorídrico. Em um estudo que destaca a importância de avaliar os materiais quando os mesmos se encontram em função e não apenas de forma individual, Magne \& Douglas (1999), observam que, os materiais odontológicos devem copiar ao máximo a estrutura dos dentes naturais em função e estética. Analisando o esmalte dentário desprendido da dentina, o mesmo se torna extremamente friável, porém quando unido à dentina pela junção amelo-dentinária tanto a dentina quanto o esmalte formam uma estrutura única que resiste as mais adversas situações na cavidade bucal.

As cerâmicas apresentam cor, textura e propriedades mecânicas superiores às das resinas compostas, e por isso têm sido o material de escolha para devolver a estética em dentes anteriores. As porcelanas são consideradas imbatíveis no quesito características de cor e translucidez dos dentes naturais, e há ainda a vantagem de serem um material friável, que não sofrem deformação plástica sob estresse. E com o advento dos materiais e a técnica adesiva de cimentação, obteve-se um aumento significativo de resistência à fratura desse material (CONCEIÇÃO, 2007).Assim, o desenvolvimento de novos materiais e técnicas tem sido fundamental para se alcançar correções e/ou alterações estéticas. A busca constante pela naturalidade dos procedimentos restauradores tem contribuído, expressivamente, para o desenvolvimento de melhorias nos materiais e técnicas restauradoras (CARVALHEIRA et al., 2010).

Lente de contato odontológica é o nome dado aos laminados cerâmicos, por comparação da espessura das lentes de contato oftalmológicas, o que requer desgastes mínimos da estrutura dental, ou mesmo eliminam totalmente a necessidade desses desgastes. Fato que agrada a maioria dos pacientes que, embora exijam uma estética 
melhor de seus sorrisos, não aceitam desgastar demasiadamente seus dentes para ganhar espaço necessário para o laminado cerâmico na face vestibular dos dentes, sem criar sobre contorno (FRANCCI et al., 2011). Os laminados cerâmicos são indicadas em situações que, a estrutura ou posição dental permite acréscimos de material, como aumento da borda incisal, aumento de volume vestibular, fechamento de diastemas, abfrações e retrações gengivais até restaurações oclusais para aumento da dimensão vertical, desde que não modifiquem ou criem um sobrecontorno (CONCEIÇÃO, 2007). Apesar de ter seu auge na década de 80 e meados dos anos noventa, os laminados cerâmicos estão sendo utilizados cada vez mais, como alternativa estética, por ser cada vez menos espessa e com boa resistência biomecânica (GUESS et al.2011)

Na Odontologia, a presença de alguma anormalidade nos dentes anteriores, como alteração de cor, forma, tamanho ou posição, pode afetar prejudicialmente o sorriso (ROSSATO et al., 2010). Essas alterações interferem na imagem do individuo perante a sociedade, podendo influenciar na sua vida pessoal e profissional. Hoje em dia, existe uma crescente tendência de valorização de um sorriso daquilo que se considera esteticamente agradável, o que incentiva a cada dia a busca por alternativas de tratamento que modifiquem ou melhorem a aparência do sorriso do paciente (BERNABE \& FLORES-MIR, 2007). Os laminados cerâmicos ganharam espaço devido à facilidade de acesso dos pacientes quanto às informações da mesma, e por ser uma técnica que não necessita de anestesia, além de preservar a estrutura dentária. Porém o caso deverá ser corretamente planejado e obedecer às indicações específicas para que se obtenha o sucesso (LIMA, 2013). A odontologia restauradora surge então com o objetivo o restabelecimento da estrutura dentária perdida, oferecendo assim a reabilitação com função e estética, devolvendo ao paciente sua autoestima.

\section{RELATO DE CASO}

Paciente do sexo feminino, compareceu a Clinica de Odontologia da Universidade Ceuma (UNICEUMA), tendo como queixa principal insatisfação estética de seu sorriso onde relatou haver diferença no formato e cor na região anterior nos dentes 12 e 21 devido a restaurações que considerava mal executadas, em resina 
Revista Ceuma Perspectivas, Edição Especial, V Congresso de Saúde e Bem Estar Ceuma. Vol. 30, nº2, 2017. ISSN Eletrônico: 2525-5576.

composta (Figura 1), sendo etiologia, um trauma dental. A paciente também relata que, essas restaurações já foram refeitas duas vezes, e sempre apresentam fraturas. No exame clínico intra-oral é notório na paciente a presença de caninos bastante pontiagudos, o que passa a se destacar como ponto de dominância no sorriso, desviando a atenção dos incisivos centrais para os caninos, resultando em um sorriso desarmônico. Além disso foi possível constatar a queixa inicial relatada pela paciente e observar que a mesma apresenta uma boa higiene oral, com boa saúde dental e periodontal.

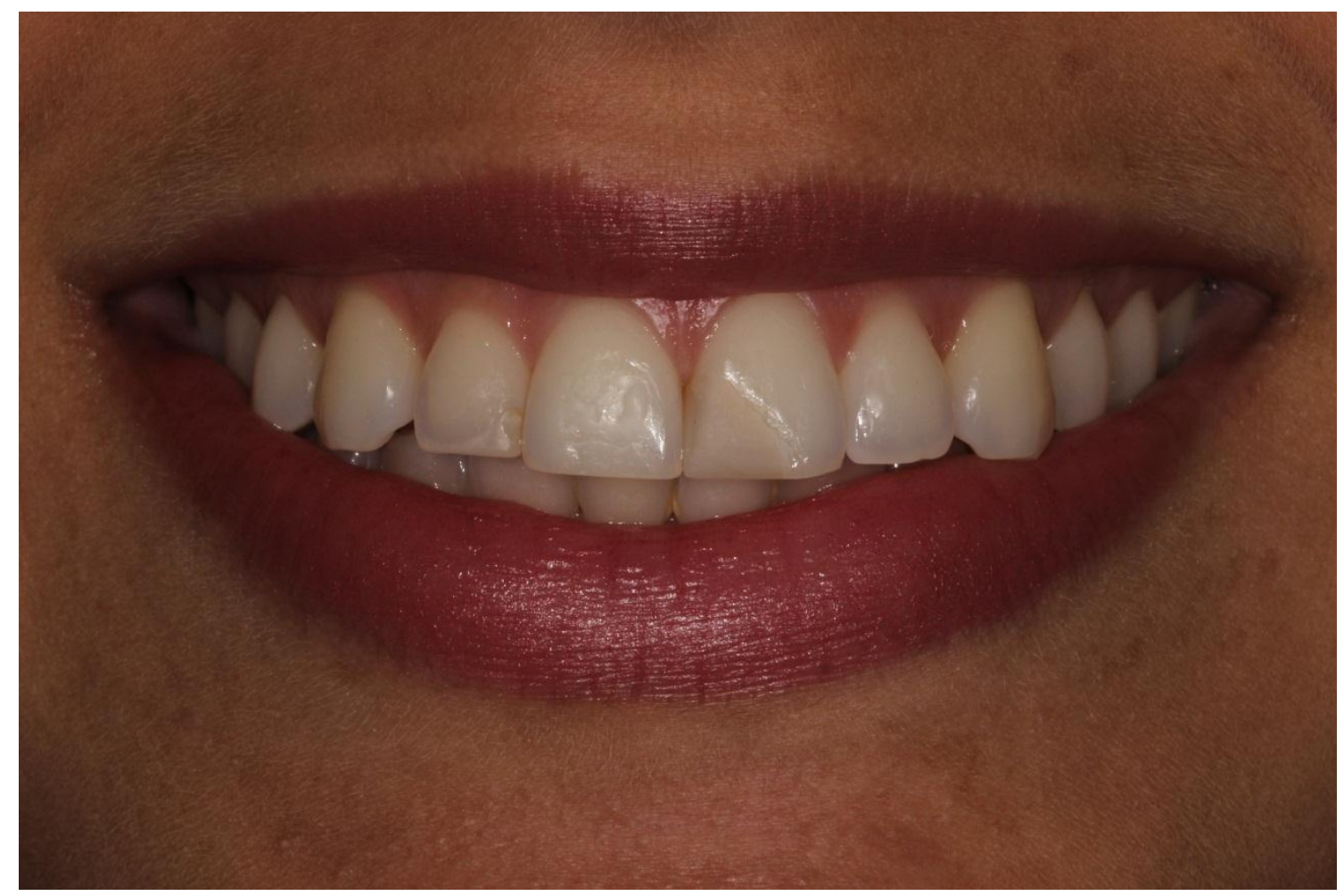

Figura 1: Fotografia inicial

Como plano de tratamento para esse caso, foi escolhido, juntamente com à paciente, a reabilitação estética com laminados cerâmicos, por preservar maior quantidade de estrutura dental e por apresentar um resultado mais próximo do natural. Foram planejados quatros laminados cerâmicos, para os incisivos centrais e laterais superiores. A paciente foi cuidadosamente informada acerca das etapas operatórias do procedimento e também sobre os cuidados que deveriam ser tomados após o tratamento, tendo, após isso, assinado um Termo de Consentimento para dar início ao tratamento. 
Revista Ceuma Perspectivas, Edição Especial, V Congresso de Saúde e Bem Estar Ceuma. Vol. 30, n02, 2017. ISSN Eletrônico: 2525-5576.

Após a definição do plano de tratamento, foi realizado protocolo fotográfico para o planejamento digital com o objetivo de melhor comunicação com o laboratório de prótese. Em seguida, foi realizada também moldagem do arco superior e inferior com moldeiras de estoque, por meio de alginato (Hydrogum $5^{\circledR}$; Zhermack ${ }^{\circledR}$ ), o molde foi vazado com gesso tipo IV (Durone IV ${ }^{\circledR}$; Dentisply ${ }^{\circledR}$ ) e o modelo de estudo foi enviado para o laboratório para que fosse realizado enceramento diagnóstico. Este, por sua vez, serve para que o paciente tenha previsibilidade do seu caso, e para demonstrar as alterações propostas no tratamento.

Após o intercurso de quinze dias, o enceramento diagnóstico foi apresentado à paciente, que, ao demonstrar reação positiva ao mesmo, seguiu-se a realização do Mock-up (Figura 2). A partir do enceramento diagnóstico foi confeccionado um molde com polivinil siloxano $\left(3 \mathrm{M} / \mathrm{ESPE}^{\circledR}\right)$ e em seguida realizado o mock up utilizando resina bis-acrílica $\left(3 \mathrm{M} / \mathrm{ESPE}^{\circledR}\right)$. O tempo de presa do material foi aguardado, os excessos foram removidos e o ajuste oclusal e estético realizados, ficando assim registrado a reprodução do enceramento diagnóstico, evidenciando à paciente o possível aspecto final da reabilitação proposta. O brilho da resina bis-acrílica foi obtido por meio de fricção de álcool 70\%. A paciente foi orientada a utilizar o mock-up por uma semana para que pudesse ter a certeza que era aquele o resultado esperado a ser obtido. Transcorrido o prazo de 7 dias, a paciente retornou à clínica alegando estar muito satisfeita com o resultado do Mock-up. 


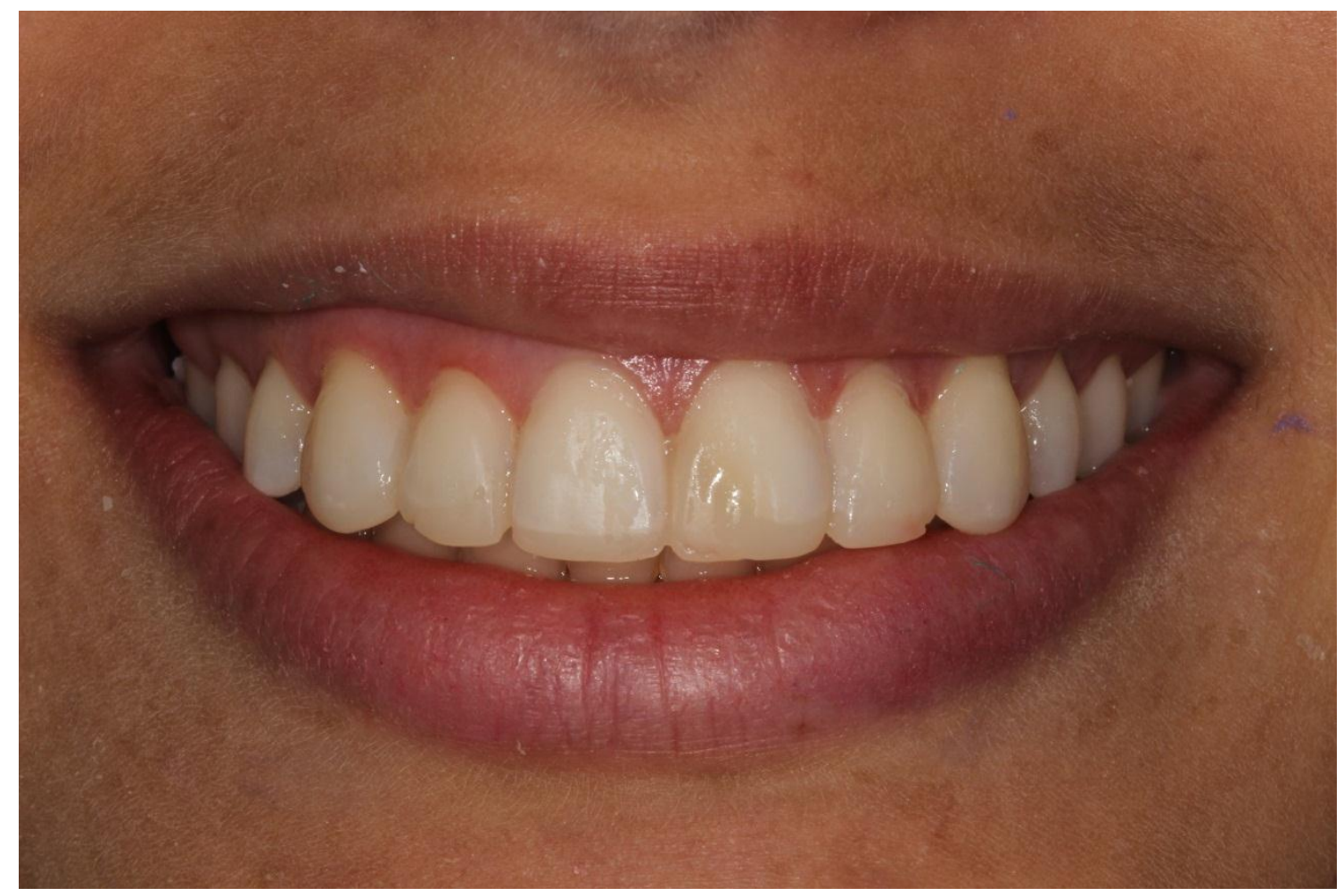

Figura 2: Mock-up

Dando continuidade ao tratamento, foi realizada confecção dos preparos minimamente invasivos nos dentes 12, 11, 21 e 22 para receber os quatro laminados cerâmicos, com pontas diamantadas (KG SORENSEN $\left.{ }^{\circledR}\right)$ \#1014 para delimitação do termino cervical e \#3215 para desgaste na face vestibular, remoção dos ângulos vivos e áreas retentivas melhorando assim, o eixo de inserção e adaptação para os laminados cerâmicos. Os preparos, foram mantidos a nível de esmalte e o acabamento foi feito com disco de lixa Sof-Lex ${ }^{\circledR}$ Pop On.

Ao final dos preparos, foram feitos novos registros fotográficos dos dentes preparados (Figura 3), e os fio de afastamento gengival (Figura 4) foram inseridos, inicialmente o fio três zeros $(000)$ e depois o fio dois zeros $(00)$ (Retractor - Roeko ${ }^{\circledR}$ ) para realização de nova moldagem pela técnica de dupla impressão, com moldeira de estoque e polivinil siloxano $\left(3 \mathrm{M} / \mathrm{ESPE}^{\circledR}\right)$. Inicialmente, a moldagem foi feita apenas com a pasta pesada, à medida que o último fio inserido, fez-se sua remoção e a pasta leve era colocada sobre o dente e, logo após, o molde com a pasta pesada foi reposicionado, obtendo assim o molde dos dentes preparados (Figura 5). A paciente 
Revista Ceuma Perspectivas, Edição Especial, V Congresso de Saúde e Bem Estar Ceuma. Vol. 30, nº2, 2017. ISSN Eletrônico: 2525-5576.

recebeu novamente o Mock-up de forma provisória, até que os laminados estivessem finalizados após envio ao laboratório.

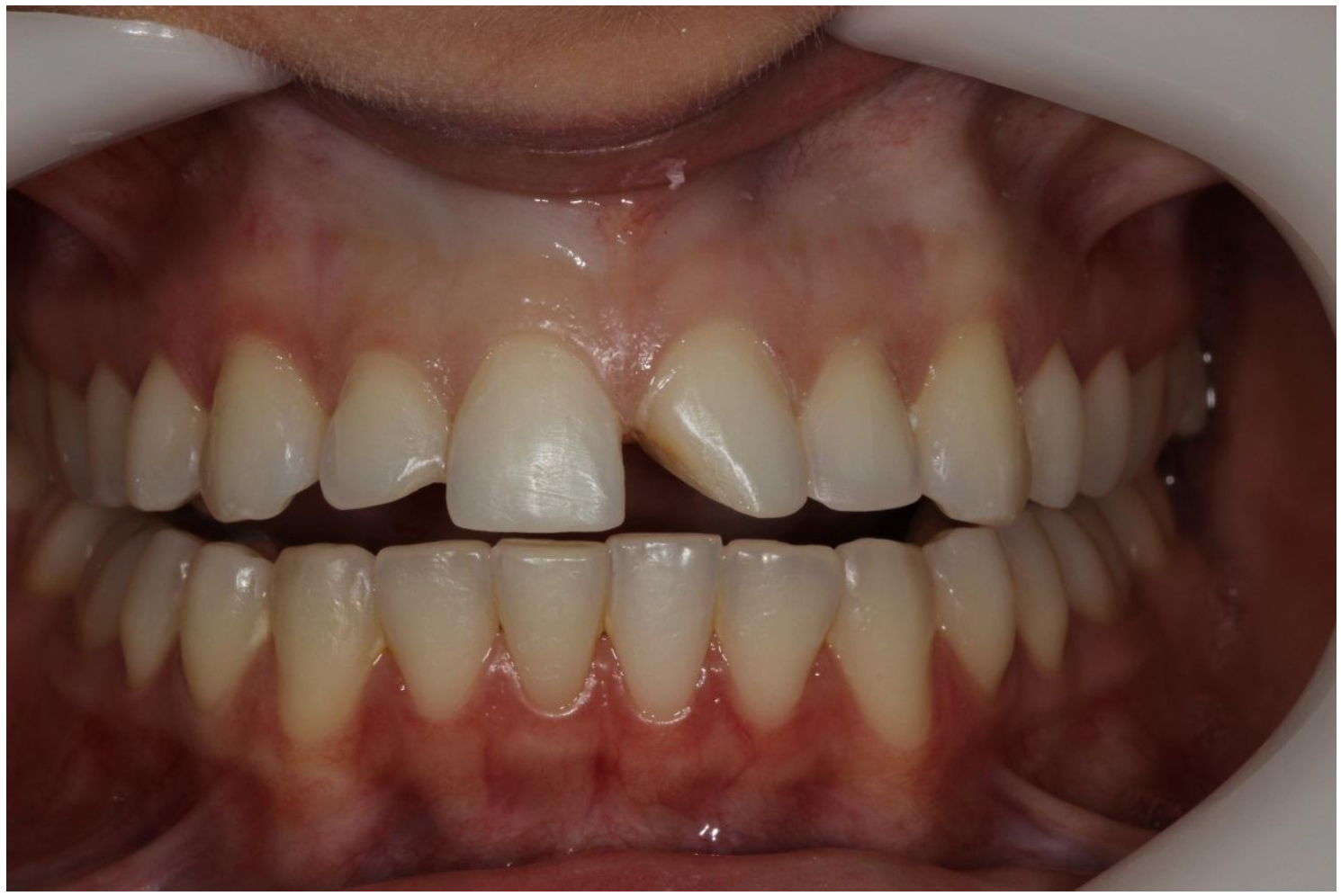

Figura 3: Dentes 12, 11, 21,22 preparados

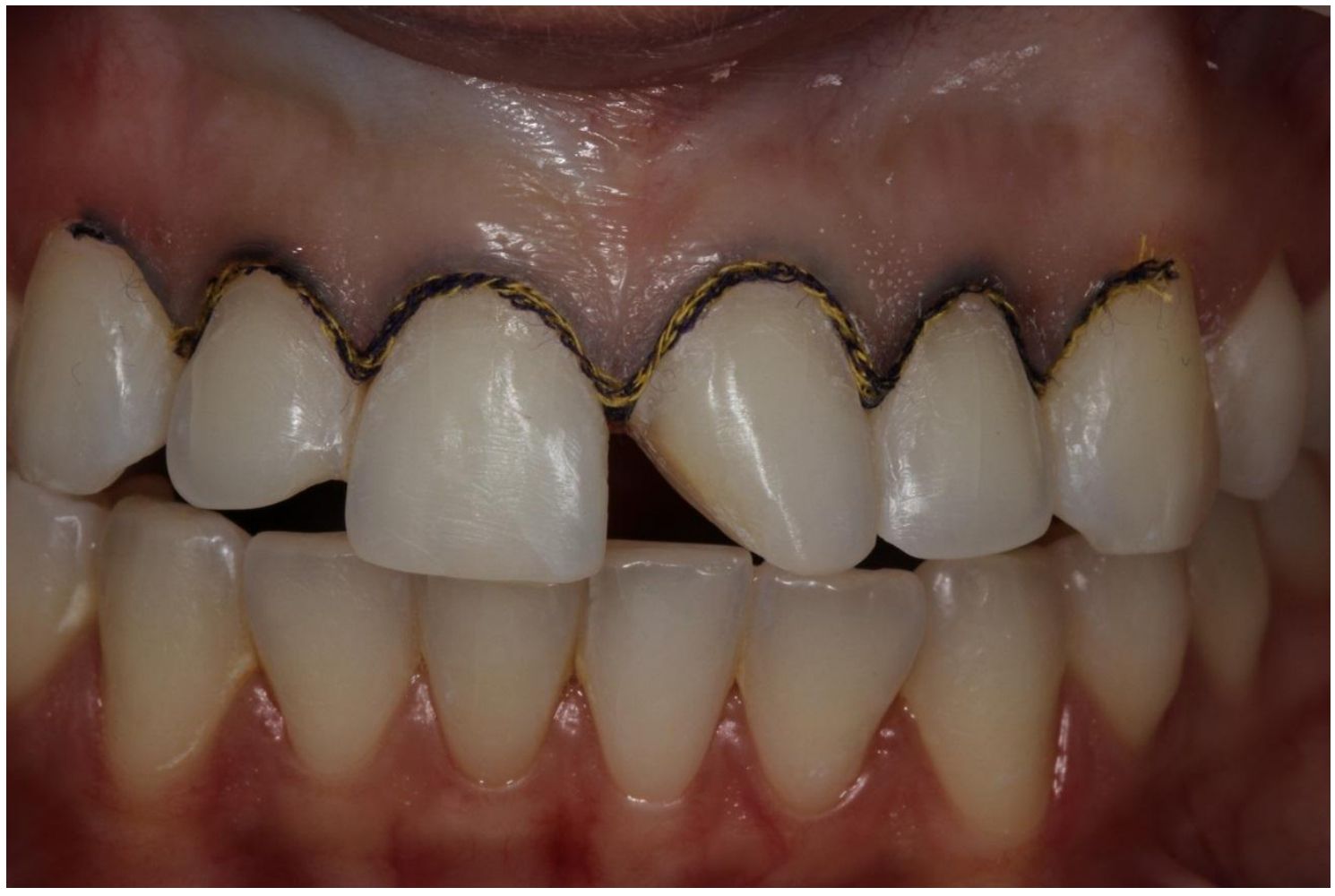

Figura 4: inserção dos fios retratores 000 e 00. 


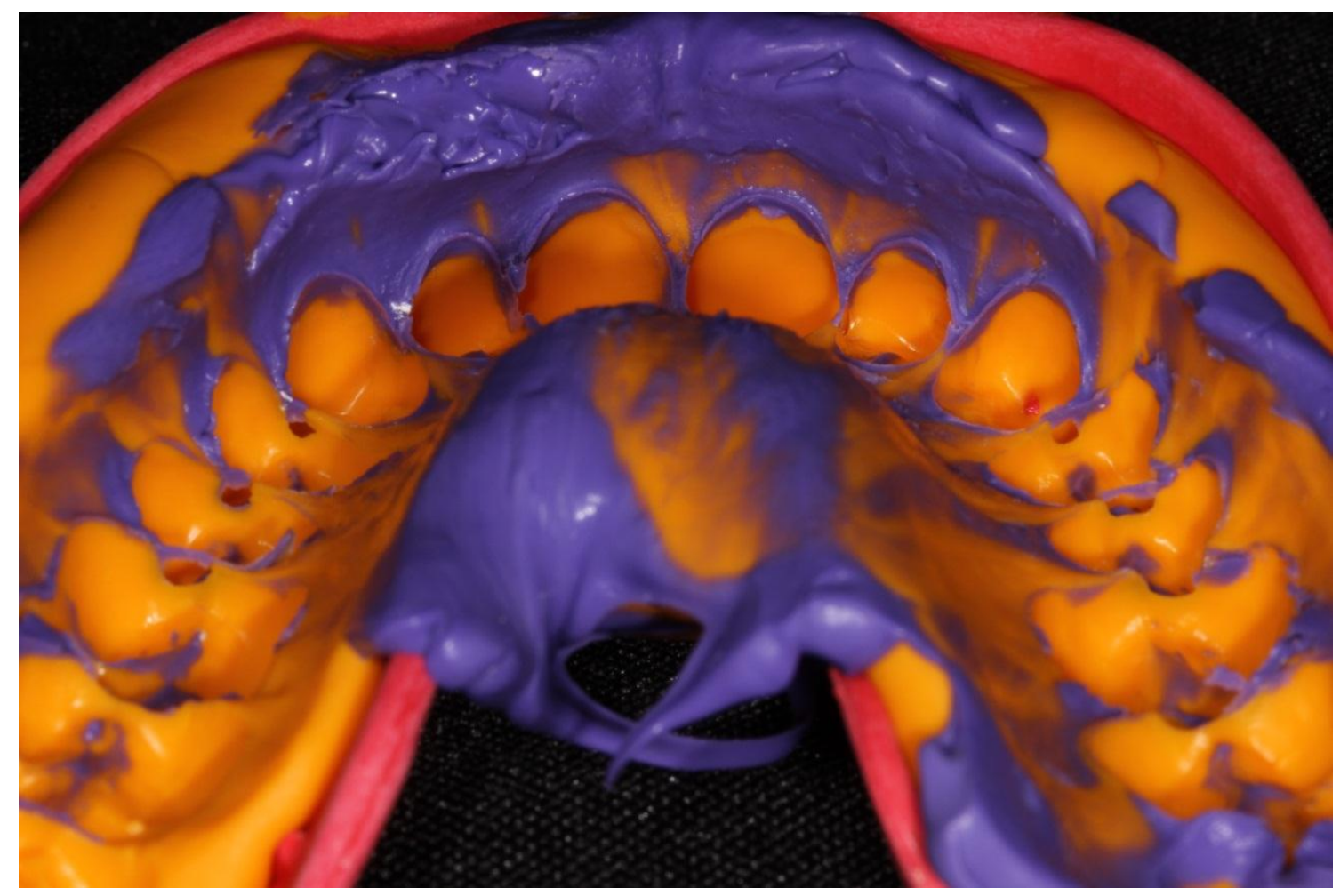

Figura 5: Molde dos dentes preparados

Os laminados cerâmicos foram confeccionados em cerâmica vítrea à base de dissilicato de lítio (IPS E.max, IvoclarVivadent ${ }^{\circledR}$ ) cor B1, a cerâmica foi maqueada e depois levada ao forno para incorporação dos pigmentos. O protocolo fotográfico foi enviado ao laboratório ao laboratório juntamente com a moldagem para melhor comunicação com o ceramista, permitindo a ele conhecer as características e detalhes do sorriso da paciente.

Após quinze dias a paciente retornou a clinica, o provisório foi removido, os laminados foram testados previamente. Para a seleção da cor do cimento resinoso, realizou-se o teste com as pastas de prova "Try-in", que foram dispensadas na face interna dos laminados cerâmicos e estes posicionados sobre os dentes preparados Após a simulação com as pastas de prova e aprovação do paciente, foi selecionado o cimento da cor transparente.

O isolamento relativo foi realizado por meio de abridor de boca e sugador. Iniciou-se a hibridização do substrato dental, para receber os laminados com ácido fosfórico $35 \%$ (Ultradente ${ }^{\circledR}$ ) por 30 segundos, seguidos de lavagem com jato de ar/água, 
Revista Ceuma Perspectivas, Edição Especial, V Congresso de Saúde e Bem Estar Ceuma. Vol. 30, n02, 2017. ISSN Eletrônico: 2525-5576.

secagem, e então foi aplicado o adesivo (Adper Single Bond $2-3 \mathrm{M}^{\circledR}$ ), removido o excesso com um leve jato de ar e fotopolimerizado por 20 segundos cada preparo. O protocolo de tratamento interno dos laminados cerâmicos também foi realizado com ácido fluorídrico 10\% (Condac Porcelana $^{\circledR}$ ) por 20 segundos, lavagem, com jato de ar/água por 60 segundos, secagem e aplicação do agente de união silano (Prosil $\mathrm{FGM}^{\circledR}$ ) por 1 minuto. O cimento resinoso fotopolimerizavel (Variolink Esthetic LC Ivoclar Vivadent ${ }^{\circledR}$ ) foi inserido em cada peça e posicionado sobre os preparos. Para remoção cuidadosa dos excessos de cimento da cervical e proximais, foi feita a fotopolimerização com unidade de LED por aproximadamente 5 segundos, para que o material enrijecesse um pouco, e somente a partir disso, com o auxilio de uma sonda exploradora e um microbush, os excessos foram removidos, e realizada a fotopolimerização com unidade de LED final de 60 segundos em cada dente, 30 segundos na face vestibular e 30 segundos na face palatina. Por fim foram feitos os registros fotográficos finais (Figura 6). A paciente demonstrou grande satisfação com o resultado final e foi orientada quanto à importância de manutenção de uma boa saúde bucal e também quanto à sua dieta.

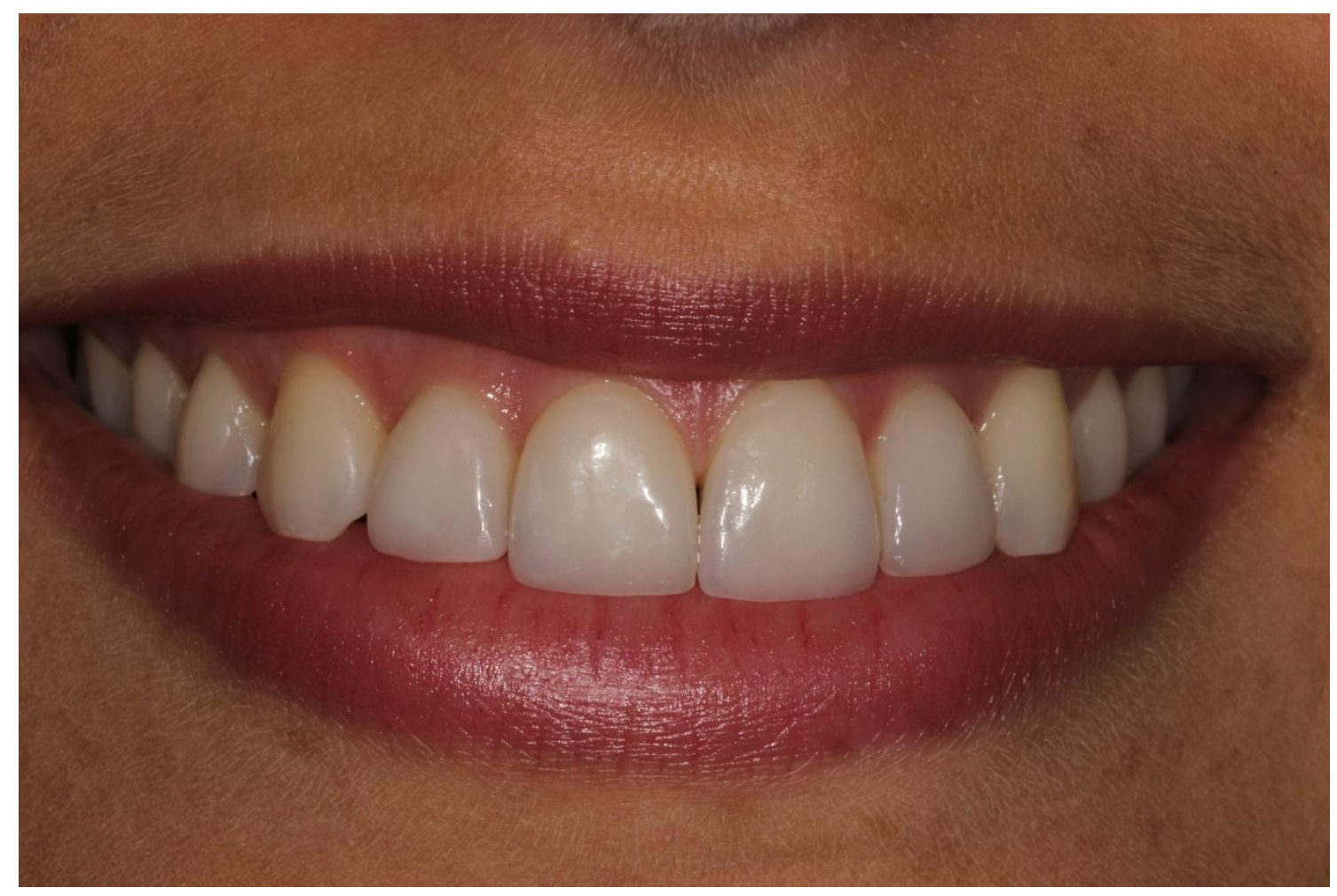

Figura 6: fotografia final após cimentação dos laminados 


\section{DISCUSSÃO}

Atualmente, o principal objetivo da Odontologia é a obtenção de restaurações que preencham requisitos estéticos, mecânicos e funcionais realizados dentro de uma abordagem minimamente invasiva para os tecidos dentários (JORDAN, 2015). Atuando dentro desta abordagem, os laminados cerâmicos tornaram-se populares para a reabilitação estética dos dentes anteriores (ALMEIRA JR et al., 2015).

Esta técnica se baseia em um desgaste dentário mais conservador quando comparado às coroas dentais cerâmicas, além de demostrar excelente biocompatibilidade com o tecido gengival (PEUMANS et al., 1998; PIPPIN et al., 1995). Em geral, o desgaste dentário necessário para este tipo de tratamento não ultrapassa a camada de esmalte, variando de 0,5 a $1,0 \mathrm{~mm}$ de espessura. Consequentemente, os laminados cerâmicos são finos e relativamente translúcidos (HORN, 1983; TURGUT \& BAGIS, 2013).

A translucidez dos laminados torna o processo de seleção de cor uma etapa difícil de ser executada. Considerando que, quanto mais finos são os laminados, maior será a passagem e dispersão de luz e a estrutura dental subjacente exercerá uma influência significativa sobre a cor final da restauração (TURGUT \& BAGIS, 2013). A cor, espessura e grau de translucidez da cerâmica (DAVIS et al., 1992; CHU et al., 2007), a cor e espessura do agente de cimentação definitiva (YAMAN et al., 1997; JOYNER, 2004), bem como o processo de envelhecimento do sistema de cimentação adesiva (AZEVEDO et al., 2011; KAMPOUROPOULOS et al., 2014) também são fatores que podem afetar a cor final da restauração a curto ou a longo prazo.

Do ponto de vista estético, é essencial obter a aprovação do paciente antes da realização de qualquer procedimento definitivo, como o processo de cimentação de laminados cerâmicos (TASCHNER et al., 2009). No entanto, pelos diversos fatores descritos, percebe-se que ainda é um desafio para os dentistas combinar as propriedades ópticas dos dentes naturais com as dos elementos cerâmicos na composição de um efeito harmonioso (ALGHZALI et al., 2010; KAMPOUROPOULOS et al., 2014). Várias abordagens têm sido descritas para prever e/ou ajustar a cor final das restaurações (TURGUT \& BAGIS, 2013; XING et al., 2010). 
Recentemente, os fabricantes introduziram no mercado sistemas de cimentação específicos para laminados cerâmicos, os chamados sistemas veneers. Os sistemas veneers incluem agentes de cimentação fotoativados em diversas opções de cores e pastas hidrossolúveis em cores correspondentes a destes cimentos, as chamadas pastas try-in (DELLA BONA \& NORTHEAST SE, 1994; XING et al., 2010). Por serem disponibilizados em uma variedade de cores, os cimentos resinosos poderiam ser utilizados na tentativa de modificar a cor final dos laminados cerâmicos (BALDERAMOS, 1997; DELLA BONA \& NORTHEAST SE, 1994). Além disso, as pastas try-in serviriam como indicadores da cor final a ser obtida, servindo como um guia de escolha do cimento resinoso tanto para o dentista quanto para o paciente (WANG et al., 1999; KARAAGACLIOGLU \& YILMA, 2008).

Santos et al., (2014), afirmam que, antes do profissional realizar o plano de tratamento, o mesmo deve ter conhecimento das características, propriedades e limitações do material, ter domínio das técnicas operatórias, para se obter um resultado seguro. Kacker et al., (2011), salientaram que a falha no diagnóstico e planejamento pode levar a resultados indesejados, como dentes proeminentes, com sobrecontorno, monocromáticos e artificiais.

De acordo com essas premissas, foram tomados os cuidados necessários para o sucesso desse caso. O preparo foi restrito a esmalte, com exceção do dente 21 , que apresentara uma fratura que compreendia a face vestibular, mesial e palatina. $\mathrm{O}$ termino dos preparos se restringiram ao nível supra gengival e para que não houvesse erro na escolha da cor do cimento resinoso, foi utilizada a pasta try-in.

\section{CONCLUSÃO}

Com a finalização do caso foi possível observar que os laminados cerâmicos apresentam excelentes resultados clínicos. Para que não houvesse erro na escolha da cor do cimento resinoso, foi utilizada a pasta try-in, como pasta teste, por apresentar três graus diferentes de luminosidade, (baixo, médio, alto), e ainda três intensidades para os níveis baixo e alto, podendo, dessa forma, maximizar os resultados ópticos.

Concluimos ainda que, com preparos minimamente invasivos e laminados cerâmicos, é possível devolver a estética e função ao paciente. Porem é necessário que 
seja feito um correto diagnóstico, planejamento e ter domínios das técnicas operatórias, para que o resultado seja satisfatório

\section{REFERÊNCIAS}

ALGHAZALI N, LAUKNER J, BURNSIDE G, JARAD FD, SMITH PW, PRESTON AJ. An investigation into the effect of try-in pastes, uncured and cured resin cements on the overall color of ceramic veneer restorations: an in vitro study. J Dent 38 Suppl 2: e78-86, 2010.

ALMEIDA JR, SCHMITT GU, KAIZER MR, BOSCATO N, MORAES RR. Resin- based luting agents and color stability of bonded ceramic veneers. J Prosthet Dent 114(2):272-7, 2015.

AZEVEDO CUBAS GB, CAMACHO GB, DEMARCO FF, PERREIRA-CENCI T. The Effect of Luting Agents and Ceramic Thickness on the Color Variation of Different Ceramics against a Chromatic Background. Eur J Dent 5(3):245-52, 2011.

BALDERAMOS LP, O'KEEFE KL, POWERS JM. Color accuracy of resin cements and try-in pastes. Int J Prosthodont 10(2):111-5, 1997.

BARATIERI et al., Soluções clínicas: fundamentos e técnicas. São José: Editora Ponto, 2008.

BERNABE, E; FLORES MIR, C. Influence of anterior occlusal chacateristics onself-perceived dental appearance in Young adults. Angle Orthod. 77:5; 831-36; 2007.

BUONOCORE, MG. A simple method of increasing the adhesion 1. of acrylic filling materials to enamel surfaces. J Dent Res, 34(6):849-53;1955.

CARDOSO, P.C., DECURSIO, R.A., LOPES, L.G., SOUZA, J.B., A importância da Pasta de Prova (Try-in) na Cimentação de Facetas Cerâmicas - Relato de Caso; 2011.

CHU FC, CHOW TW, CHAI J. Contrast ratios and masking ability of three types of ceramic veneers. J Prosthet Dent 98(5):359-64, 2007.

DAVIS BK, AQUILINO SA, LUND PS, DIAZ-ARNOLD AM, DENEHY GE. Colorimetric evaluation of the effect of porcelain opacity on the resultant color of porcelain veneers. Int $\mathrm{J}$ Prosthodont 5(2):130-6, 1992.

DELLA BONA A, NORTHEAST SE. Shear bond strength of resin bonded ceramic after different try-in procedures. J Dent 22(2):103-7, 1994.

HEKIMOGLU C, ANIL N \& ETIKAN I. Effect of accelerated aging on the color stability of cemented laminate veneers International Journal of Prosthodontics. 13:29-33, 2000.

HONG G, MURATA H, LI Y, SADAMORI S, HAMADA T. Influence of denture cleansers on the color stability of three types of denture base acrylic resin. J Prosthet Dent 101:205-13, 2009.

HORN HR. Porcelain laminate veneers bonded to etched enamel. Dent Clin North Am 27(4):671-84, 1983.

JANDA R, ROULET J-F, LATTA M, KAMINSKY M, RUTTERMANN St. Effect of exponential 
polymerization on color stability of resin-based filling materials. Dent Mater. 23:696-704, 2007.

JOINER A. Tooyh colour: a review of the literature. J Dent 32:3-12, 2004.

JORDAN A. Clinical aspects of porcelain laminate veneers: considerations in treatment planning and preparation design. J Calif Dent Assoc 43(4):199-202, 2015.

JUNIOR, R.A., Facetas estéticas; Cerâmica ou resina? Do planejamento ao resultado. Florianopólis: Universidade Federal de Santa Catarina; 2016.

KAMPOUROPOULOS D, GAINTANTZOPOULOU M, PAPAZOGLOU E, KAKABOURA A. Colour matching of composite resin cements with their corresponding try-in pastes. Eur J Prosthodont Restor Dent 22(2):84-8, 2014

KARAAGACLIOGLU L, YILMA B. Influence of cement shade and water storage on the final color of leucite-reinforced ceramics. Oper Dent 33(4):386-91, 2008.

KUCUKESMEN HC, USUMEZ A, OZTURK N, EROGLU E. Change of shade by light polymerization in a resin cement polymerized beneath a ceramic restoration. $J$ Dent, 36:219-223, 2008.

LIMA, P. Laminados cerâmicos minimamente invasivos: Uma revisão sobre lentes de contatos dentais. Trabalho de conclusão de curso; 2013.

LIMA, R.D.W., FIGUEIREDO, J. R. A., ANDRADE, A.K.M., DUARTE, R.M., Otimizando a Estética do Sorriso através de Coroa Cerâmica "Metal Free" - Relato de caso. Revista brasileira de ciência da saúde. p165-170; 2013.

LINDEN JJ, SWIFT JR EJ, BOYER DB, DAVIS BK. Photo-activation of resin cements through porcelain veneers. J Dent Res. 70:154-157, 1991.

OKIDA, R.C., VIEIRA, W.S.C., RAHAL, V., OKIDA, D.S.S. Lentes de contato: Restaurações minimamente invasivas na solução de problemas estéticos; 2016.

PARAVINA RD, ONTIVEROS JC \& POWER JM. Accelerated aging effects on color and translucency of bleaching-shade composites. J of Esthetic and Restorative Dentistry. 16:117-126, 2004.

PEUMANS M, VAN MEERBEEK B, LAMBRECHTS P, VUYLSTEKE-WAUTERS M, VANHERLE G. Five-year clinical performance of porcelain veneers. Quintessence Int 29(4):211-21, 1998.

PIPPIN D, MIXSON JM, SOLDAN-ELS AP. Clinical evaluation of restored maxillary incisors: veneers vs. PFM crowns. J Am Dent Assoc 126(11):1523-9, 1995.

POLYZOIS GL, YANNIKAKIS SA, ZISSIS AJ, DEMETRIOU PP. Color changes of denture base material after disinfection and sterilization immersion. Int J. Prosthodont. 10:83-9, 1997.

ROSSATO, D.M., SAADE. E. G., SAADE. J. R. C., PORTO-NETO. S. T. Coroas estéticas anteriores em cerâmica metal-free: relato de caso clínico; 2010.

SAMRA AP, PEREIRA SK, DELGADO LC, BORGES CP. Color stability evaluation of aesthetic restorative materials. Bra Oral Res. 22:205-10, 2008. 
Revista Ceuma Perspectivas, Edição Especial, V Congresso de Saúde e Bem Estar Ceuma. Vol. 30, nº2, 2017.

ISSN Eletrônico: 2525-5576.

SCHULZE KA, MARSHALL SJ, GANSKY SA, MARSHALL GW. Color stability and hardness in dental composites after accelerated aging. Dent Mater 19: 612-9, 2003.

TASCHNER M, FRANKENBERGER R, GARCÍA-GODOY F, ROSENBUSCH S, PETSCHELT A, KRAMER N. IPS Empress inlays luted with a self-adhesive resin cement after 1 year. Am $J$ Dent 22(1):55-9, 2009.

TERZIOGLU H, YILMAZ B, YURDUKORU B. The effect of different shades of specific luting agents and IPS empress ceramic thickness on overall color. Int $J$ Periodontics Restorative Dent 29(5):499-505, 2009.

TURGUT S, BAGIS B. Effect of resin cement and ceramic thickness on final color of laminate veneers: an in vitro study. J Prosthet Dent 109(3):179- 86, 2013.

WANG X, POWERS JM. Color differences between a resin cement and try- in paste. Zhonghua Kou Qiang Yi Xue Za Zhi 34(1):58-9, 1999.

XING W, JIANG T, MA X, LIANG S, WANG Z, SA Y, WANG Y. Evaluation of the esthetic effect of resin cements and try-in pastes on ceromer veneers. J Dent 38 Suppl 2:e87-94, 2010.

YAMAN, P., S. R. QAZI. Effect of adding opaque porcelain on the final color of porcelain laminates. J Prosthet Dent 77(2):136-40, 1997. 